\title{
Concordancia entre el diagnóstico presuntivo y tomográfico en canes con enfermedades encefálicas y de columna vertebral
}

\section{Concordance between presumptive and tomographic diagnosis in dogs with encephalic and spinal diseases}

\author{
Eben Salinas C..,2, Néstor Falcón P.3,4
}

\section{Resumen}

\begin{abstract}
El objetivo del estudio fue determinar la concordancia entre el diagnóstico clínico y tomográfico en canes con enfermedades en encéfalo y columna vertebral. Se realizó un estudio observacional retrospectivo analítico, evaluándose 196 historias clínicas e informes tomográficos de clínicas veterinarias de Lima Metropolitana durante el periodo 20092015. Se incluyeron en el estudio 76 tomografías encefálicas y 120 de la columna vertebral. Del total de pacientes, 113 fueron machos (58\%) y 83 hembras (42\%) y 120 casos $(61.2 \%)$ correspondieron a razas definidas. Las principales enfermedades diagnosticadas clínicamente y por tomografía computarizada (TC) fueron: hernia toracolumbar, epilepsia canina, hidrocefalia y hernia cervical. Se relacionó el diagnóstico clínico con la presencia de una patología diagnosticada por TC mediante el índice de kappa, clasificándose la concordancia como pobre $(\mathrm{k}=0.38)$. Se concluye que el diagnóstico clínico presuntivo y la TC tienen muy baja concordancia al diagnosticar una enfermedad o lesión en encéfalo o medula espinal.
\end{abstract}

Palabras clave: hernia discal, tomografía computarizada, diagnostico presuntivo, perros

\section{AbSTRACT}

The aim of this study was to determine the concordance between the clinical and tomographic diagnosis in dogs with brain and spine illnesses. An analytical retrospective observational study was carried out, evaluating 196 clinical histories and tomographic

\footnotetext{
${ }^{1}$ Universidad Cientifica del Sur, Lima, Perú

${ }^{2}$ E-mail: esalinasc@ucientifica.edu.pe

${ }^{3}$ Laboratorio de Epidemiología y Salud Pública en Veterinaria, Facultad de Medicina Veterinaria y Zootecnia, Universidad Peruana Cayetano Heredia, Lima, Perú

${ }^{4}$ E-mail: nestorfalcon@upch.pe
}

Recibido: 13 de septiembre de 2019

Aceptado para publicación; 4 de junio de 2020

Publicado: 11 de agosto de 2020 
reports of veterinary clinics in Metropolitan Lima during the period 2009-2015. This included 76 brain and 120 spinal tomography scans. Of the total of patients, 113 were males (58\%) and 83 females (42\%) and 120 cases $(61.2 \%)$ corresponded to purebreds. The main diseases diagnosed clinically and by computed tomography (CT) were thoracolumbar hernia, canine epilepsy, hydrocephalus, and cervical hernia. The clinical diagnosis was related to the presence of a pathology diagnosed by CT using the kappa index and the concordance was classified as poor $(\mathrm{k}=0.38)$. It is concluded that the presumptive clinical diagnosis and CT have very low concordance when diagnosing a disease or injury in the brain or spinal cord.

Key words: herniated disc, computerized tomography, presumptive diagnosis, dogs

\section{INTRODUCCIÓN}

Las enfermedades y lesiones del sistema nervioso conforman un conjunto de patologías de diagnóstico complejo, donde es preponderante evaluar clínicamente al paciente en búsqueda de signos característicos que dirijan el diagnóstico. En el proceso de la evaluación del paciente neurológico, el diagnóstico presuntivo se construirá a partir de la anamnesis, el examen físico y el examen neurológico. Los hallazgos clínicos, en la mayoría de los casos, requerirán confirmación mediante técnicas imagenológicas o métodos diagnósticos invasivos con el objetivo de obtener el diagnóstico definitivo.

El examen neurológico debe ser precedido por un completo examen físico general con el fin de excluir problemas ajenos a los neurológicos, pero con signos clínicos similares como: dolor, convulsión, paresia y parálisis (Pellegrino, 2014). La anamnesis permitirá, entre otras, identificar el tipo de presentación de la patología, si esta es aguda o crónica, progresiva o estática, persistente o intermitente (De Lahunta y Glass, 2015).

Para el examen neurológico (EN) Pellegrino (2014) plantea nueve etapas correlativas para el manejo de un paciente con un problema neurológico: (1) Establecer el motivo principal de la consulta neurológica; (2) Determinar las características, curso y la evolución de los problemas mediante la reseña y anamnesis; (3) El EN propiamente dicho; (4) Determinar el sitio de la lesión planteando el diagnóstico neuroanatómico; (5) Plantear un diagnóstico presuntivo, el cual implica realizar una categorización del trastorno sobre la base de los datos relacionados en su inicio y progresión, utilizando además los conocimientos de las probables enfermedades y procesos mórbidos descritos en la bibliografía y la experiencia clínica; (6) Construir un diagnóstico diferencial; (7) Elegir los métodos de diagnóstico complementarios más sensibles; (8) Plantear el tratamiento más acorde a los hallazgos clínicos; y (9) Brindar un pronóstico.

Los exámenes complementarios son de uso frecuente para confirmar o rechazar el diagnóstico presuntivo. En el paciente con afección neurológica, las radiografías tienen la función de realizar una exploración general en búsqueda de cambios en los tejidos; sin embargo, es dependiente de la radio densidad de los tejidos y de la superposición de los tejidos circundantes. Con el uso de la Tomografía Computarizada (TC) y Resonancia Magnética (RM) se ha logrado una mayor precisión en el diagnóstico definitivo. La TC es una técnica de diagnóstico por imagen, inmediato y no invasivo, que utiliza rayos X para generar imágenes de distintos planos anatómicos (axiales, sagitales o coronales). Esto se logra a través de cortes milimétricos transversales al eje cefalocaudal 
(Lim et al., 2010; De Lahunta y Glass, 2015). La TC puede establecer una imagen directa de los cambios patológicos, debido al gran contraste de sus imágenes. La TC permite discriminar diferencias de densidad radiológica del $0.05 \%$ frente al $0.5 \%$ de la radiología convencional (Ruoff et al., 2018). Esta técnica tiene grandes ventajas sobre la técnica de rayos $\mathrm{X}$ convencional, pues permite una diferenciación superior de los tejidos blandos y evita la superposición de estructuras subyacentes (Aguinaga et al., 2006); sin embargo, podría presentar deficiencias en identificar lesiones profundas del cerebro, tronco encefálico y parénquima medular, debido principalmente a su baja afinidad por los tejidos blandos o pobremente calcificados (Dennis, 2003).

Antes de recomendar el uso de la TC (u otra técnica diagnóstica), se requiere que la enfermedad neurológica sea abordada de forma adecuada y precisa. La TC no sustituye a la anamnesis, ni la exploración general y neurológica. Por tanto, el abordaje clínico del paciente garantizará que el estudio se realice en la zona de interés y la imagen diagnóstica sea compatible con los antecedentes del paciente (Israel et al., 2009).

El objetivo del presente trabajo fue determinar el grado de concordancia entre los diagnósticos presuntivos y los hallazgos tomográficos en pacientes caninos con patologías encefálicas y de columna vertebral. Esta información contribuye a conocer si los resultados obtenidos por el examen clínico y tomográfico son equivalentes o intercambiables como métodos diagnósticos de lesiones y enfermedades en encéfalo y medula espinal.

\section{Materiales y Métodos}

El estudio comprendió 196 historias clínicas e informes tomográficos correspondientes a pacientes caninos con alteraciones en encéfalo y columna vertebral, obtenidos entre 2009 y 2015 en clínicas veterinarias de
Cuadro 1. Clasificación cualitativa de los valores kappa como grado de acuerdo más allá del azar ${ }^{1}$

\begin{tabular}{cc}
\hline Valores de K & Interpretación \\
\hline $0-0.20$ & Deficiente \\
$0.21-0.40$ & Pobre \\
$0.41-0.60$ & Aceptable \\
$0.61-0.80$ & Buena \\
$0.81-1.00$ & Excelente \\
\hline
\end{tabular}

${ }^{1}$ Abraira (2000)

Lima Metropolitana. Los diagnósticos clínicos se obtuvieron de reportes e historias clínicas de cinco médicos veterinarios titulados y con especialidad en medicina de animales de compañía. Las TC y los diagnósticos

Cuadro 2. Características demográficas de 196 pacientes caninos sometidos a examen neurológico y tomografía de cráneo y columna vertebral (Lima, 2009-2015)

\begin{tabular}{lcc}
\hline $\begin{array}{l}\text { Características } \\
\text { demográficas }\end{array}$ & $\mathrm{n}$ & $\%$ \\
\hline Sexo & 113 & 57.7 \\
$\quad$ Macho & 83 & 42.3 \\
Hembra & & \\
Estrato etario & 12 & 6.1 \\
$\quad$ Cachorros $<1$ año & 108 & 55.1 \\
$\quad$ Adultos $1-7$ años & 16 & 38.8 \\
$\quad$ Geronte $>7$ años & 76 & \\
Raza & 120 & 61.2 \\
$\quad \begin{array}{l}\text { Definida } \\
\text { No definida }\end{array}$ & 76 & 38.8 \\
Tamaño & & \\
Miniatura y & 69 & 35.2 \\
$\quad$ pequeño & & \\
Mediano & 83 & 42.3 \\
Grande & 44 & 22.5 \\
\hline
\end{tabular}


Cuadro 3. Diagnósticos clínicos presuntivos de encéfalo y columna vertebral de 196 pacientes caninos (Lima, 20092015)

\begin{tabular}{lcc}
\hline $\begin{array}{c}\text { Diagnóstico clínico } \\
\text { presuntivo }\end{array}$ & $\mathrm{n}$ & $\%$ \\
\hline Hernia toracolumbar & 73 & 37.2 \\
Epilepsia & 21 & 10.7 \\
Hernia cervical & 20 & 10.2 \\
Encefalopatía tumoral & 16 & 8.2 \\
Hernia lumbosacra & 12 & 6.1 \\
Hidrocefalia & 11 & 5.6 \\
Hernia lumbar & 9 & 4.6 \\
Contusión cerebral & 7 & 3.6 \\
Síndrome vestibular & 5 & 2.6 \\
Otros ${ }^{1}$ & 22 & 11.1 \\
\hline Total & 196 & 100 \\
\hline${ }^{1}$ Accidente cerebro vascular, encefalopatía \\
infecciosa, fractura de columna cervical, \\
tumor en cráneo, fractura lumbar, \\
encefalopatía degenerativa, fractura en \\
columna toracolumbar, fractura de cráneo, \\
encefalopatía tóxica
\end{tabular}

tomográficos fueron realizados por el centro de diagnóstico por imágenes Resocentro, el cual brinda apoyo diagnostico por más de 10 años a los médicos veterinarios de Lima, Perú. Las tomografías fueron realizadas con un tomógrafo de segunda generación Siemens Somaton Spirit Dual Slice CT Scanner (dos cortes).

Se recolectaron los siguientes datos: características demográficas (sexo, estrato etario, raza y tamaño); ubicación de la lesión (cerebro y columna vertebral), descripción y tipo de lesión y diagnóstico tomográfico. Los datos fueron procesados y analizados con el software SPSS v. 19.0. Los análisis estadísticos se realizaron de forma independiente, segmentando la base de acuerdo con la región anatómica (cerebro, columna vertebral). Los resultados del diagnóstico clínico y TC fueron resumidos según las variables demográficas consideradas en el estudio. Se relacionó el diagnóstico clínico presuntivo con la presencia de una patología diagnosticada por TC mediante el índice de kappa y se utilizó una escala para expresar cualitativamente la fuerza de concordancia (Cuadro 1) (Abraira, 2000).

\section{Resultados}

De los 196 historias clínicas e informes tomográficos correspondientes a pacientes con alteraciones de encéfalo y columna vertebral, 76 casos $(38.8 \%)$ correspondieron a

Cuadro 4. Diagnósticos tomográficos de enfermedades de encéfalo y columna vertebral de 196 pacientes caninos (Lima, 2009-2015)

\begin{tabular}{|c|c|c|}
\hline $\begin{array}{l}\text { Diagnósticos } \\
\text { tomográficos }\end{array}$ & $\mathrm{n}$ & $\%$ \\
\hline Normal & 59 & 30.1 \\
\hline Hernia toracolumbar & 51 & 26 \\
\hline Hidrocefalia & 16 & 8.2 \\
\hline Hernia cervical & 15 & 7.7 \\
\hline Hernia lumbar & 11 & 5.6 \\
\hline Hernia lumbosacra & 8 & 4.1 \\
\hline Encefalopatía tumoral & 7 & 3.6 \\
\hline Edema cerebral & 5 & 2.6 \\
\hline Discoespondilosis & 4 & 2 \\
\hline Otros $^{1}$ & 20 & 10 \\
\hline Total & 196 & 100 \\
\hline \multicolumn{3}{|c|}{$\begin{array}{l}1 \text { Contusión cerebral, Isquemia cerebral, } \\
\text { Tumor de cráneo, Fractura lumbar, Fractura } \\
\text { de columna cervical, Fractura de columna } \\
\text { toracolumbar, Neoplasia vertebral, Neopla- } \\
\text { sia medular, Hemivértebra, Hematoma } \\
\text { cerebral, Infarto cerebral, Hipertensión } \\
\text { endocraneana, Encefalopatía degenerativa }\end{array}$} \\
\hline
\end{tabular}


Cuadro 5. Resultados de la concordancia entre diagnósticos presuntivos y tomográficos en 196 pacientes caninos con enfermedades en encéfalo y columna vertebral (Lima, 2009-2015)

\begin{tabular}{|c|c|c|c|c|c|c|c|c|c|c|c|c|c|c|c|c|c|c|c|c|c|c|c|}
\hline & & \multicolumn{22}{|c|}{ Diagnóstico tomográfico $^{1}$} \\
\hline Pax & $\operatorname{Diax}^{2}$ & $\mathrm{Nr}$ & HTL & $\mathrm{H}$ & $\mathrm{HC}$ & $\mathrm{HL}$ & HLS & ET & EC & $\mathrm{DE}$ & $\overline{\mathrm{CC}}$ & IC & $\mathrm{TC}$ & $\mathrm{FL}$ & FCC & FTL & $\mathrm{NV}$ & $\mathrm{NM}$ & $\mathrm{HV}$ & HMC & IFC & HEC & ECD \\
\hline 73 & HTL & 13 & 47 & & 1 & 4 & 2 & & & 4 & & & & & & & 1 & & 1 & & & & \\
\hline 21 & $\mathrm{E}$ & 16 & & 3 & & & & 1 & & & & 1 & & & & & & & & & & & \\
\hline 20 & $\mathrm{HC}$ & 5 & & & 14 & & & & & & & & & & & & & 1 & & & & & \\
\hline 16 & ET & 4 & & 4 & & & & 5 & 1 & & & & & & & & & & & & 1 & & 1 \\
\hline 12 & HLS & 2 & 1 & & & 4 & 5 & & & & & & & & & & & & & & & & \\
\hline 11 & $\mathrm{H}$ & 3 & & 6 & & & & & 1 & & & 1 & & & & & & & & & & & \\
\hline 9 & HL & 2 & 3 & & & 3 & 1 & & & & & & & & & & & & & & & & \\
\hline 7 & $\mathrm{CC}$ & 3 & & 1 & & & & & 1 & & 2 & & & & & & & & & & & & \\
\hline 5 & $\mathrm{ACV}$ & 3 & & & & & & & 1 & & & 1 & & & & & & & & & & & \\
\hline 5 & SV & & & \begin{tabular}{|l|}
2 \\
\end{tabular} & & & & & 1 & & 1 & & & & & & & & & & & 1 & \\
\hline 4 & $\mathrm{EF}$ & 4 & & & & & & & & & & & & & & & & & & & & & \\
\hline 3 & $\mathrm{FCC}$ & 2 & & & & & & & & & & & & & 1 & & & & & & & & \\
\hline 3 & $\mathrm{TC}$ & & & & & & & & & & & & 3 & & & & & & & & & & \\
\hline 2 & FL & & & & & & & & & & & & & 2 & & & & & & & & & \\
\hline 2 & ED & 1 & & & & & & 1 & & & & & & & & & & & & & & & \\
\hline 1 & FTL & & & & & & & & & & & & & & & 1 & & & & & & & \\
\hline 1 & $\mathrm{FC}$ & & & & & & & & & & & & & & & & & & & 1 & & & \\
\hline 1 & ET & 1 & & & & & & & & & & & & & & & & & & & & & \\
\hline 196 & Total & 59 & 51 & 16 & 15 & 11 & 8 & 7 & 5 & 4 & 3 & 3 & 3 & 2 & 1 & 1 & 1 & 1 & 1 & 1 & 1 & 1 & 1 \\
\hline
\end{tabular}

${ }^{1} \mathrm{Nr}=$ normal, $\mathrm{EC}=$ edema cerebral, $\mathrm{DE}=$ disco espondilosis, $\mathrm{CC}=$ contusión cerebral, $\mathrm{IC}=$ isquemia cerebral, $\mathrm{FL}=$ fractura lumbar, NV = neoplasia vertebral, $\mathrm{NM}=$ neoplasia medular, $\mathrm{HV}=$ hemivértebra, $\mathrm{HMC}=$ hematoma cerebral, IFC = infarto cerebral, $\mathrm{HEC}=$ hipertensión endocraneana, $E C D$ = encefalopatía degenerativa

${ }^{2}$ Diagnóstico presuntivo: $\mathrm{HTL}=$ hernia toracolumbar, $\mathrm{E}=$ epilepsia, $\mathrm{HC}=$ hernia cervical, $\mathrm{ET}=$ encefalopatía tumoral, $\mathrm{HLS}=$ hernia lumbosacra, $\mathrm{H}=$ hidrocefalia, $\mathrm{HL}=$ hernia lumbar, $\mathrm{CC}=$ contusión cerebral, $\mathrm{ACV}=$ accidente cerebro vascular, $\mathrm{SV}=$ síndrome vestibular, $\mathrm{EF}=$ encefalopatía infecciosa, $\mathrm{FCC}=$ fractura de columna cervical, $\mathrm{TC}=$ tumor de cráneo, $\mathrm{FL}=$ fractura lumbar, ED = encefalopatía degenerativa, $\mathrm{FTL}=$ fractura toracolumbar, $\mathrm{FC}=$ fractura de cráneo, ET = encefalopatía toxica

TC encefálica y $120(61.2 \%)$ a columna vertebral; 113 pacientes fueron machos $(58 \%)$ y 86 hembras (44\%); y 120 casos $(61.2 \%)$ correspondieron a razas definidas. El estrato etario y el tamaño más frecuente fueron el adulto y mediano con $108(55.1 \%)$ y 83 (42.3\%) casos, respectivamente (Cuadro 2).
Los diagnósticos clínicos más frecuentes correspondieron a hernia toracolumbar con 73 casos (37.2\%), epilepsia con 21 casos (10.7\%), hernia cervical con 20 casos (10.2\%), encefalopatía tumoral con 16 casos $(8.2 \%)$ y hernia lumbosacra con 12 casos (6.1\%) (Cuadro 3). 
Los diagnósticos tomográficos más frecuentes fueron la hernia toracolumbar con 51 casos $(26 \%)$, hidrocefalia con 16 casos (8.2\%), hernia cervical con 15 casos $(7.7 \%)$, hernia lumbar con 11 casos $(5.6 \%)$ y hernia lumbosacra con 8 casos $(4.1 \%)$. Los casos reportados por tomografía como normales correspondieron a 59 casos (30.1\%) (Cuadro 4).

En el Cuadro 5 se presentan los datos cruzados correspondientes a los diagnósticos presuntivos con los obtenidos por TC, mostrando la concordancia entre ambos diagnósticos para cada paciente. Hubo concordancia en 137 pacientes en ambos métodos de diagnóstico con una concordancia observada de $81 \%$. El índice de Kappa como grado de acuerdo más allá del azar fue de 0.38 . Teniendo como estándar de diagnóstico clínico el examen neurológico, la TC mostró una sensibilidad de $56 \%$ y especificidad de $17.1 \%$, con un valor predictivo positivo de $37 \%$ y valor predictivo negativo de $31 \%$.

\section{Discusión}

El proceso del diagnóstico neurológico se sustenta en un adecuado examen clínico y en el apoyo en los exámenes complementarios. Puede ser muy complicado lograr el diagnóstico definitivo o etiológico en pacientes afectado por una enfermedad o lesión en el sistema nervioso. Esta complicación en la corroboración del diagnóstico clínico puede ser a consecuencia de la escasa disponibilidad de algunas herramientas diagnósticas (TC o RM), así como por factores económicos de parte de los propietarios (Platt y Olby, 2014).

Se obtuvo una concordancia pobre entre los diagnósticos presuntivos y sus respectivos estudios tomográficos. La baja concordancia se pudo dar a consecuencia de errores en los diagnósticos clínicos presuntivos planteados luego del examen neurológico. Dentro de las causas relacionadas a los errores diagnósticos y que influyen en la toma de decisiones clínicas se incluyen las distracciones, la fatiga y el estrés de la carga de trabajo (Graber, 2013), así como la poca pericia e inexperiencia del médico tratante (Cortés, 2005), la cual puede influenciar negativamente en el diagnóstico definitivo y tratamiento a efectuar.

La baja concordancia también se puede sustentar en las desventajas del uso de la TC para algunas estructuras del encéfalo y columna vertebral. Dennis (2003) señala para este punto una baja sensibilidad para las lesiones vasculares e inflamatorias y baja calidad de contraste de los tejidos blandos. Adicionalmente se sabe que la imagen tomográfica de la fosa caudal del cráneo (cerebelo y tronco encefálico) puede ser poco definida, debido al artefacto de endurecimiento del haz (Porat-Mosenco et al., 2004). Si bien el objetivo del estudio no fue determinar si la TC es el mejor método para confirmar el diagnóstico clínico, este hallazgo indica que los resultados obtenidos por el examen clínico y tomográfico no pueden ser equivalentes o intercambiables como métodos diagnósticos de lesiones y enfermedades en encéfalo y medula espinal.

En cuanto a los hallazgos en el presente estudio, las hernias de disco toracolumbares (HTL) representaron los diagnósticos clínicos presuntivos y tomográficos más frecuentes. Al realizarse el estudio tomográfico a los 73 pacientes con diagnóstico presuntivo de HT se pudo observar que la concordancia con el diagnóstico dado por la TC no fue muy alta (47/73). Esto coincide con lo reportado por Israel et al. (2009), quienes no lograron destacar a la TC en el diagnóstico de la HTL por sobre otros métodos radiológicos como la mielografía, que solo obtuvieron una sensibilidad relativa del $80 \%$. Esto indica que ante una sospecha de HTL, el médico veterinario tratante puede decidir entre ambas modalidades para confirmar el diagnóstico (Israel et al., 2009). Asimismo, Cooper et al. (2014) al comparar la TC con la RM para el diagnóstico de la HTL reportaron una mayor sensibilidad relativa de la RM (98.5\%). Además, 
en este mismo estudio, la RM pudo lograr la diferenciación entre una extrusión versus una protrusión del disco

La epilepsia canina fue el segundo diagnóstico presuntivo más frecuente con $21 \mathrm{ca}-$ sos. En estos pacientes, el estudio tomográfico reportó diagnósticos de hidrocefalia, encefalopatía tumoral e isquemia cerebral. El objetivo de los estudios complementarios y por imágenes en el paciente epiléptico, es lograr la ubicación del foco epileptógeno o la lesión estructural que cause las convulsiones (De Risio et al., 2015). En ese sentido, si bien la $\mathrm{TC}$ es recomendada para estudios rápidos y para identificar estructuras en base a su grado de mineralización, el consenso médico indica dirigir el proceso diagnóstico a herramientas como el electroencefalograma (EEG), estudio del líquido cefalorraquídeo o la RM (Bush et al., 2002). En los seres humanos, el EEG es considerado como el método de diagnóstico gold standard, pues contribuye con la clasificación y tipo de convulsiones. Al comparar el aporte de la TC con el EEG, González-Velázquez y Juárez-Mesinas (2008) en un estudio en niños con diagnóstico de epilepsia encontraron una concordancia muy baja $(\mathrm{k}=0.095)$ entre ambos métodos para el hallazgo de lesiones cerebrales relacionadas con la localización del foco epileptógeno.

En el presente estudio, la hidrocefalia (HI) fue diagnosticada clínicamente en 11 casos y confirmada tomográficamente en seis casos. En el paciente con diagnóstico presuntivo de $\mathrm{HI}$ es imprescindible la confirmación diagnóstica, lo cual permitirá implementar el tratamiento oportuno para evitar el incremento de la presión intracraneana. Se puede considerar a la TC como una herramienta de diagnóstico complementaria y adecuada para la HI. Sin embargo, la RM de bajo campo predomina en la investigación de la HI, ya que es un método sensible, que produce imágenes de alta calidad con artefactos mínimos (Robertson 2011; Vaquero et al., 2011). La RM también resultó más sensible que la TC para identificar pequeñas lesiones focales en la fosa caudal en perros hidrocefálicos (Thomas, 2010). En el campo clínico, el médico debe desarrollar la capacidad de decidir por el método complementario para la confirmación diagnóstica de la HI. Los pacientes afectados con HI suelen presentar signos a muy temprana edad, por lo que el uso de la TC puede ser negativo dado el uso de radiación ionizante; sin embargo, se debe preferir la TC cuando la anestesia del paciente no debe ser prolongada o no pueda realizarse (Biel et al., 2013).

La sensibilidad de la TC, tomando como prueba gold standard al examen neurológico fue de $56 \%$, representando una baja capacidad de la prueba para detectar la enfermedad en sujetos enfermos. Cabe destacar que no hay informes disponibles sobre la sensibilidad diagnóstica de la TC en muchas enfermedades neurológicas en caninos. Principalmente, se ha reportado una sensibilidad relativa de la TC para detectar una hernia discal del 88.6\% (79.5 - 94.2\%) (Cooper, 2014). Cabe destacar, que estas conclusiones se lograron al utilizar tomógrafos de reciente generación o helicoidales, que a diferencia del utilizado en el presente trabajo (segunda generación), pudo mejorar sustancialmente la sensibilidad.

No se dispone de estudios que evalúen la sensibilidad de la TC en grandes poblaciones de canes. Las principales publicaciones son de casos clínicos o estudios primarios, en los cuales no se ha logrado destacar la ventaja de la TC sobre otros métodos de diagnóstico (estudio del líquido cefalorraquídeo, EEG, RM). Sin embargo, los resultados parecen indicar un mayor aporte de la RM para identificar lesiones cerebrales (Gielen et al., 2013), así como una herramienta confiable para el pronóstico de supervivencia en pacientes con lesión cerebral traumática (Beltran et al., 2014).

Una limitación para el desarrollo del estudio ha sido la escasa información disponible sobre el tema. En Medicina Veterinaria, no es una práctica frecuente realizar estu- 
dios que valoren la concordancia entre diagnóstico clínico y los resultados de los exámenes para ayuda diagnóstica. Esta situación contrasta con Medicina Humana, donde se realizan prácticamente de manera rutinaria, estudios de concordancia inter o intra evaluador para medir la confiabilidad o reproducibilidad de las evaluaciones (González-Velázquez et al., 2008; CampoArias y Herazo, 2010; Goicochea et al., 2015).

\section{Conclusiones}

- Existe una concordancia pobre entre el diagnóstico clínico presuntivo y tomográfico en canes con enfermedades de encéfalo y columna vertebral.

- Los resultados obtenidos por el examen clínico y tomográfico no pueden ser equivalentes o intercambiables como métodos diagnósticos de lesiones y enfermedades en encéfalo y medula espinal.

- La decisión de usar la tomografía se debe basar en un criterio técnico y clínico. Es imprescindible conocer las ventajas y desventajas de esta técnica en las diferentes estructuras del encéfalo y columna vertebral.

\section{Literatura Citada}

1. Abraira V. 2000. El índice kappa. Semergen 27: 247- 249.

2. Aguinaga F, Rivera J, Tamayo L. 2006. Tomografía axial computarizada y resonancia magnética para la elaboración de un atlas de anatomía segmentaria a partir de criosecciones axiales del perro. Rev Colomb Cienc Pecu 19: 451-459.

3. Beltran E, Platt SR, Mcconnell JF, Dennis R, Keys DA, De Risio L. 2014. Prognostic value of early magnetic resonance imaging in dogs after traumatic brain injury: 50 Cases. J Vet Internal Med 28: 1256-1262.
4. Biel M, Kramer M, Forterre F, Jurina K, Lautersack O, Failing K, Schmidt M. 2013. Outcome of ventriculo peritoneal shunt implantation for treatment of congenital internal hydrocephalus in dogs and cats: 36 cases (2001-2009). J Am Vet Med Assoc 242: 948-958. doi: 10.2460/javma.242.7.948

5. Bush WW, Barr CS, Darrin EW, Shofer FS, Vite CH, Steinberg SA. 2002. Results of cerebrospinal fluid analysis, neurologic examination findings, and age at the onset of seizures as predictors for results of magnetic resonance imaging of the brain in dogs examined because of seizures: 115 cases (19922000). J Am Vet Med Assoc 220: 781784. doi: 10.2460 /javma.2002.-220.781

6. Campo-Arias A, Herazo E. 2010. Concordancia intra - e interevaluadores. Rev Colomb Psiquiatr 39: 424-432.

7. Cooper J, Young B, Griffin J, Fosgate G, Levine J. 2014. Comparison between noncontrast computed tomography and magnetic resonance imaging for detection and characterization of thoracolumbar myelopathy caused by intervertebral disk herniation in dogs. Vet Radiol Ultrasound 55: 182-189. doi: 10.1111/vru. 12114

8. Cortés G 2005. El error médico en pediatría. CONAMED 11(4): 76-78.

9. De Lahunta A, Glass E, Kent M. 2015. Veterinary neuroanatomy and clinical neurology. $3^{\mathrm{a}}$ ed. St Louis, USA: Saunders. $600 \mathrm{p}$.

10. De Risio L, Bhatti S, Muñana K, Penderis J, Stein V, Tipold A, Berendt $M$, et al. 2015. International veterinary epilepsy task force consensus proposal: diagnostic approach to epilepsy in dogs. BMC Vet Res 11: 148.

11. Dennis $R$. 2003. Advanced imaging: indications for $\mathrm{CT}$ and MRI in veterinary patients. In Practice 25: 243-255. doi: 10.1136/inpract.25.5.243

12. Higgs J, Jones MA, Loftus S, Christensen N. 2008. Clinical reasoning in the health professions. $3^{\mathrm{a}}$ ed. Amsterdam: Butterworth Heinemann. 504 p. 
13. Gielen I, Kromhout K, Gavin P, Van Ham L, Polis I, Van Bree H. 2013. Agreement between low-field MRI and CT for the detection of suspected intracranial lesions in dogs and cats. $\mathrm{J}$ Am Vet Med Assoc 243: 367-375. doi: 10.2460/javma.243.3.367

14. Goicochea V, Oyola A, Quispe M, Muñoz Z. 2015. Concordancia entre el diagnóstico clínico presuntivo y el diagnóstico tomográfico abdomino-pélvico. Rev Méd Panacea 5(2). doi: 10.35563/ rmp.v5i2.61

15. González-Velázquez, F, JuárezMesinas DF. 2008. Concordancia electroence-falográfica tomográfica en pacientes pediátricos con epilepsia. Rev Med Inst Mex Seguro Soc 46: 261-266

16. Graber ML. 2013. The incidence of diagnostic error in medicine. BMJ Qual Saf22: ii21-ii27. doi: 10.1136/bmjqs-2012001615

17. Israel S, Levine J, Kerwin S, Levine G, Fosgate G. 2009. The relative sensitivity of computed tomography and myelography for identification of thoracolumbar intervertebral disk herniations in dogs. Vet Radiol Ultrasound 50: 247-252. doi: 10.1111/j.1740-8261.2009.01528.x

18. Lim C, Kweon OK, Choi MC, Choi J, Yoon J. 2010. Computed tomographic characteristics of acute thoracolumbar intervertebral disc disease in dogs. J Vet Sci 11: 73-79. doi: 10.4142/jvs.2010.11.1.73
19. Pellegrino F. 2014. Las claves del diagnóstico neurológico para el veterinario clínico. Argentina: Inter-Médica. $456 \mathrm{p}$.

20. Platt SR, Olby NJ. 2014. BSAVA Manual of canine and feline neurology. $4^{\text {th }}$ ed. British Small Animal Veterinary Association. $542 \mathrm{p}$.

21. Porat-Mosenco Y, Schwarz T, Kass $P H .2004$. Thick-section reformatting of thinly collimated computed tomography for reduction of skull-base-related artifacts in dogs and horses. Vet Radiol Ultrasound 45: 131-135. doi: 10.1111/j.17408261.2004.04021.x

22. Robertson I. 2011. Optimal magnetic resonance imaging of the brain. Vet Radiol Ultrasound 52: S15-S22. doi: 10.1111/j.1740-8261.2010.01781.x

23. Ruoff CM, Kerwin SC, Taylor AR. 2018. Diagnostic imaging of discospondylitis. Vet Clin North Am Small Anim Pract 48: 85-94. doi: 10.1016/ j.cvsm.2017.08.007

24. Thomas WB. 2010. Hydrocephalus in dogs and cats. Vet Clin North Am Small Anim Pract 40: 143-159. doi: 10.1016/ j.cvsm.2009.09.008

25. Vaquero PM, Ronaldo C, Da Costa, WM. 2014. Comparison of noncontrast computed tomography and high-field magnetic resonance imaging in the evaluation of great danes with cervical spondylomyelopathy. Vet Radiol Ultrasound 55: 496-505. doi: 10.1111/ vru. 12148 